\title{
AUTHORING MIXED REALITY - A COMPONENT AND FRAMEWORK-BASED APPROACH
}

\author{
Ralf Dörner ${ }^{1}$, Christian Geiger ${ }^{2}$, Michael Haller ${ }^{3}$, and Volker Paelke ${ }^{4}$ \\ ${ }^{I}$ Fraunhofer AGC, Frankfurt, Germany, ${ }^{2}$ Hochschule Harz, Harz, Germany, ${ }^{3}$ Fachhochschule \\ Hagenberg, Hagenberg, Austria, ${ }^{4} C$-LAB, Paderborn, Germany \\ doerner@agc.fhg.de,cgeiger@hs-harz.de,haller@fh-hagenberg.at, \\ vox@c-lab.de
}

\begin{abstract}
This paper describes the basic ideas of our effort to develop a framework for the structured authoring of mixed reality (MR) applications. The overall objective of the AMIRE project is to define and implement a software system that allows content experts to easily design and implement mixed reality applications without detailed knowledge about the underlying base technologies. This requires a user centred approach for authoring in contrast to existing approaches that are primarily driven by technological features and problems of MR systems. In order to support content experts in MR design, the AMIRE framework also consist of a visual authoring tool that uses a component oriented base technology for building mixed realities. The application of reusable building blocks at different levels of granularity (MR gems, MR components) is the central idea in this project.
\end{abstract}

Key words: Mixed Reality, MR gems, MR components, MR framework, Authoring tools

\section{Introduction}

How is an application that relies on MR technology actually produced? What do we need to take into account if we do not wish to produce a demonstration or a prototype only? How does the production process of a MR application differ from a Multimedia application? What are the critical issues we need to pay attention to? What should a structured production

The original version of this chapter was revised: The copyright line was incorrect. This has been corrected. The Erratum to this chapter is available at DOI: 10.1007/978-0-387-35660-0_65 
process for MR look like? How does MR content differ from Multimedia content? What do we need to learn about MR technologies and what can be hidden in basic APIs or software parts? One prominent class of potential MR applications is the class of entertainment applications like games or amusement park attractions. If MR is to become successful in this application area the questions raised above need to be answered first.

In this paper we present our ideas and the approach we have taken to find answers for these questions.

\subsection{State of the Art in MR}

The main focus of MR research is to develop AR base technologies [8, 9, 14]. There are a number of European projects that mainly focus on developing techniques for special application domains, e.g., technical service/maintenance (ARVIKA, STAR, STAR MATE), Surgery (VRSVR), tv (PISTE) and do not focus on a structured authoring approach based on reusable components. Authoring issues are considered in projects like ART.LIVE, METAVISION, INTERFACE or CHARISMATIC but most of these projects focus on image/video based techniques or focus on human/avatar-like interaction facilities [2]. Similarly, the Japanese MR project [10], sponsored by Canon, developed the necessary base for advanced MR applications but did not consider the authoring of such applications.

Consequently employing a component-based reuse of (third-party) solutions and providing a structured design method supported by visual authoring tools gives us the opportunity to reuse the results of the mentioned projects in a user-centered authoring framework.

\subsection{Components and Frameworks}

A fundamental concept for our approach is the usage of object-oriented application frameworks $[4,7]$. In essence, this means that we have a generic skeleton application that is filled with components by using dedicated authoring tools to make a final application out of the semi-complete one. The skeleton application offers specific interfaces where a component can be plugged in. With a component, we mean a software component in the sense of the component theory [13]. A component is a building block of a software system with an explicitly defined interface designed for reusability. An advantage of this approach is that the possibility to reuse components in several applications is guaranteed. Also, the author is not only offered a set of building blocks for her application, but is given a pattern for arranging them and does not need to take care how they are expected to cooperate. 
Among the first approaches for applying component and framework concepts to computer graphics is the TBAG system [5]. TBAG is a framework that implements graphical data types on a high abstraction level in $\mathrm{C}++$. An extension to a framework that can be used by the animation author in particular is implemented in the Alice animation system [11]. Alice comprises pre-defined class libraries. Keeping the encapsulation of geometry and behavior while extending the authoring environment for creating 3D applications in general, Dörner and Grimm presented the concept of threedimensional "Beans" [3]. Another recent concept for three-dimensional components was provided by Rudolph [12] in the context of specifying X3D [6].

The work described shows that component concepts can be successfully applied to 3D computer graphics and are increasingly considered a standard approach when creating 3D graphics applications. The idea of using components for augmented reality has also been used in the DWARF framework [1]. DWARF components are very highlevel (e.g. modules for tracking, UI, application) compared to the AMIRE's approach.

\section{Approach}

The goal of our approach is to enable people, not only expert researchers, to use MR for their applications as well as to create and modify these MR applications with the support of dedicated tools that foster an efficient authoring process for MR. This is the key for a more widespread use of MR, for a transfer of MR to different application domains, for exploiting synergies between different MR methodologies, and for establishing authoring itself as a new application domain for MR. Our approach for achieving these goals relies on well-established methodologies from software engineering, namely component theory and object-oriented application frameworks. We adapt it for the specific requirements for Mixed Reality by distinguishing between MR gems, MR components, and MR framework.

\subsection{MR Gems}

Similar to existing gems collections (game programming gems, graphic gems) we are producing a MR gem collection. A gem represents an efficient solution to a specific MR. Based on a classification of necessary tasks a MR application has to solve, gems are provided.

This collection will be used in our MR framework, but can also be used in other mixed reality projects. Technically, it is not AMIRE's objective to 
develop new base technologies for mixed reality programming. Instead, AMIRE will adopt existing solutions and provide efficient means to encapsulate different solutions in a uniform way. Typically, MR gems can be reused in many different applications. For example, a "work path animation"-gem that visualizes the workflow of a special machine in an oil refinery can be reused to explain painting techniques of a famous painting in a museum.

\subsection{MR Components and MR Framework}

The MR gems in turn can be used to build application specific MR components (e.g. a valve component for the oil refinery application) as well as a MR framework that defines how MR components can communicate with each other and can be integrated in an application. The MR framework provides the according infrastructure. MR components represent solutions for particular domain specific problems and they typically combine and extend MR gems towards advanced high-level features of a MR application. For example, an object recognition gem, a path animation gem and a 3D object loader gem may be combined into a "magical lens"-component that illustrate the inner details of a real machine by providing a suitable animation of virtual machine parts. MR components feature a unified interface that easily allows to configure and combine them via the MR authoring tools using a suitable component model. With these authoring tools MR components can be easily customized by end users and compared to gems- represent heavy-weight building blocks in domain specific application scenarios.

\subsection{Authoring Tools}

The consistent use of gems and components provides the base for new design approaches in the area of advanced mixed reality applications. Through reuse, adaptation and combination of existing building blocks synergies of different previous solutions can be exploited. Visual authoring tools making use of specific authoring metaphors and tailored to the special requirements of reusable mixed reality elements guarantee short development times and thus enable new design methods for MR content creation, e.g. iterative and rapid prototyping. This allows authors to concentrate on user requirements rather than to focus on technical aspects of MR technology. User centered design will lead to a wider acceptance of MR applications. It is an important objective that the authoring tools developed employ MR methodologies, especially in order to provide the user with direct preview capabilities and not to force him to specify the application on 
an abstract level only. The authoring tools are aimed at two different user groups. First, there are authoring tools for the MR provider in order to build the MR application. Second, there are authoring tools meant for the customer in order to modify the application in a limited way or express his ideas and requirements with regards to the MR provider (e.g. change the usage of a pipeline in the oil refinery application). These tools can be built by the MR provider using MR gems, MR components and MR frameworks as building bricks. The scope of the tools have to be determined by each application and depend on the skills of the customer.

\subsection{Authoring Process}

To summarize, we envision a hierarchical authoring process with different abstraction levels that builds upon the usage of MR gems, MR components and the MR framework. After the production of MR gems, we have authors who produce MR components and we collect them in a component library. Other authors produce the MR framework for authoring and for the final applications (e.g. a computer game for edutainment purposes in a company or an entertainment application as part of an ecommerce application). These authors can start the development from scratch or can modify the existing framework.

\section{THE AMIRE PROJECT}

AMIRE (Authoring Mixed Reality) is a European funded project that will be carried out from 2002 to 2004 . The objective is to fill the gap that there are no dedicated authoring metaphors or tools for Mixed Reality available at the moment neither does there exist any dedicated authoring process. Since the idea is to enable the customer to modify and extend MR content, this is considered a key development that enables customers to communicate powerfully their ideas and collaborate using MR technology. In addition, more applications using MR could be produced and evaluated using the project results. The following is considered to be an important project result:

- best practice examples for using MR in applications

- a production process for developing and modifying MR content with design recommendations

- $\quad$ specifications of MR gems, MR components, MR frameworks and dedicated MR authoring tools along with examples how to employ them. The specifications are aimed to be partly used as a basis for MR standardisation 
- demonstrators for different application domains; one for an Austrian oil refinery and one for the Spanish Guggenheim museum.

Two completely different applications will be developed in the AMIRE project to show the flexibility and efficiency of the AMIRE approach. The first demonstrator is used as an illustration example for the description of the approach. Research organizations are invited to provide their input. Based on these experiences an actual company (that is only familiar with Computer Graphics but not with MR) will develop the second demonstrator. The problems the company has when producing the MR application will be evaluated and fed back in the AMIRE approach. In the following subsections we give a short overview of the scope of the demonstrators.

\subsection{Oil Refinery Application}

The objective of the oil refinery demonstrator is to contribute to the improvement of the learning process by developing an advanced assembly training system based on innovative concepts and new cognitive approaches. By using AMIRE's concepts, the development of such a prototype is expected to be done more quickly.

\subsection{Museum Application}

The objective of the museum application is to carry on a pilot experience, extensible to other museums, oriented to improve the current visitor's museum information systems by using mixed reality techniques. This demonstrator will provide information concerning the works of art the tourist is looking at, showing details and peculiarities. This will allow consulting more in-depth information and accessing related works or objects not available to the public.

\subsection{Feasability Studies}

The adaptation of software elements, transforming them into MR gems and combining them into an application was tested in a first feasability study. In a small application we developed an interactive illustration that explains the shape of museal artefact, an antique vase. The idea is that visitors select physical fragments of the vase and are shown how this fragment fits into the original vase. 

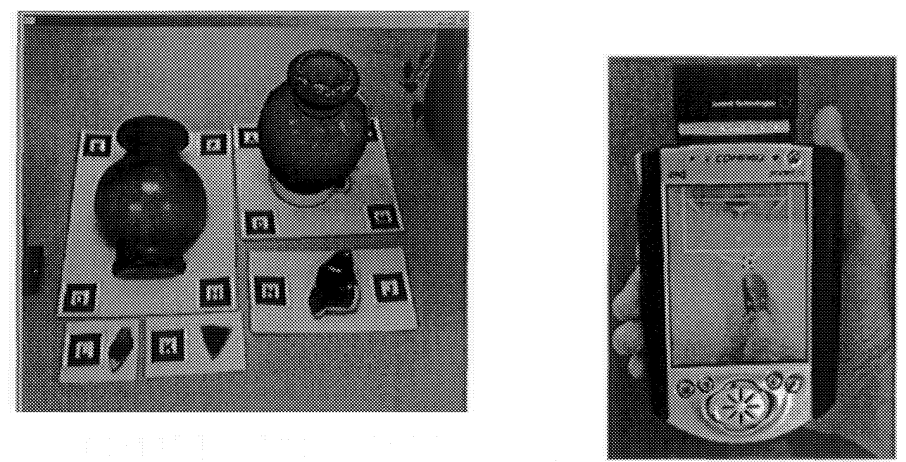

Figure 1: Feasibility Studies of Mixed Reality using the ARToolkit.

For this interactive illustration we use the concept of "virtual ghost objects", i.e., a virtual copy of the real fragment is used on an animation path to assemble the vase. Using a parametric animation path the illustration shows the correct assembling animation of a fragment independently from the fragment's start position. Physical fragments are placed on cards with markers for object tracking. The following MR elements were identified for this application:

- Path animation with parameters for start / end point

- 3D object loader for virtual fragments (OBJ format)

- Augmented reality software for object tracking and compositing The first two components were reused from our own projects and we used the ARToolkit for tracking and compositing. Figure 1 shows the results of this simple study. The user grabs a card with a mounted fragment and positions it near the artefact. The animation automatically starts by creating the ghost object and moving it from the card to the correct position at the artefact.

Another feasability study considered the design of a mobile prototype AR device for both application domains in AMIRE. Images are captured using a small camera mounted at the Compaq iPAQ. Images are encoded and sent via WirelessLan to a server (PC or Laptop) that analyses the image and augments it with computer generated content. We built a simple AR soccer game for this prototype. The user kicks the virtual ball with his real foot and tries to score a penalty goal beating a virtual goal keeper. All AR computation is currently done on a PC server running with about 10-12 frames per second. 


\section{CONCLUSION AND FUTURE WORK}

While the majority of research efforts in Mixed Reality is devoted to tackle basic problems, we described a component- and framework-based approach. This can provide efficient means to encapsulate different solutions in a uniform way. In turn, this will dramatically foster the combination of different MR methodologies in a single application and will therefore lead to new MR and imaging techniques.

In the future, we should consider revising the underlying production process, also for traditional entertainment applications. It gives us the opportunity to deal with shortcomings of the traditional approach, for instance little effort is invested in creating customized authoring environments today. The concept presented in this paper offers a more organized approach that is structured by the use of frameworks.

In the future, a discussion needs to be started how a structured authoring process and a component model and interface for Mixed Reality should be designed and especially standardized. As the applicability of MR is growing requirements need to be collected and feedback from the key players (end users, tool developers, technology and content providers) is vital for the future research efforts.

\section{ACKNOWLEDGEMENTS}

The AMIRE project is funded by the European Commission in the IST programme (IST-2001-34024). The ARSoccer prototype was realized by D. Stichling and B. Kleinjohann.

\section{REFERENCES}

[1] M. Bauer et al. Design of a Component-Based Augmented Reality Framework. Int. Symposium on Augmented Reality, ISAR 2001, New York, 29-30 October,2001

[2] http://www.cordis.lu/ist/ka4/vision/projects.htm

[3] Dörner, R., Grimm, P.: Three-dimensional Beans - creating Web content using 3D components in a 3D authoring environment. Proceedings of the Web3D-VRML 2000 fifth symposium on Virtual reality modeling language (Monterey CA, February 2000), ACM Press, pp. 69-74.

[4] Ralf Dörner, Christian Geiger, Paul Grimm, Michael Haller Authoring Tools for interactive mixed reality, Int. Conf, on Mixed Reality, Yokohama, Japan, March 200107-20

[5] Elliot, C., Schechter, R., Yeng, R., Abi-Ezzi, S.: TBAG: A High Level Framework for Interactive, Animated 3D Graphics Applications, Proceedings of Siggraph'94, pp. 421ff., ACM Press, 1994 
[6] Extensible 3D Task Group, Available at http://www.web3D.org/fs_specifications.htm

[7] Fayad, M.E., Schmidt, D., Johnson, R.: Building Application Frameworks: ObjectOriented Foundations of Framework Design, John Wiley \& Sons, New York, 1999

[8] Steven Feiner, Tobias Höllerer, Elias Gagas, Drexel Hallaway, Tachio Terauchi, Sinem Güven, and Blair MacIntyre, MARS - Mobile Augmented Reality Systems, Columbia University Computer Graphics and User Interfaces Lab, 2001

[9] Wolfgang Friedrich, ARVIKA Augmented Reality for Development, Production, and Service, Tagungsband des Informationsforum Virtuelle Produktentstehung (IVIP), May, 2000.

[10] http://www.mr-system.co.jp

[11] Pierce, J., Cobb, T., Pausch, R.: Alice, Proceedings of Siggraph'98, pp. 140ff, ACM Press, 1998

[12] Rudolph, M.: X3D Components, Web3D Consortium, Available at http://www.web3D.org/TaskGroups/x3D/lucidActual/X3Dcomponents/

[13] Sametinger, J.: Software Engineering with Reusable Components. Springer Verlag, 1997

[14] Dieter Schmalstieg, Mark Billinghurst, Ivan Poupyrev, Hirokazu Katu, AugmentedReality: The Interface is Everywhere, Course Notes of Siggraph 2001, Los Angeles, USA, August, 2001. 